EPiC Series in Computing
Volume 70, 2020, Pages 119-128
$\begin{gathered}\text { Proceedings of the 12th International Conference } \\ \text { on Bioinformatics and Computational Biology }\end{gathered}$

\title{
Co-expression networks uncover regulation of splicing and transcription markers of disease
}

\author{
Pan Zhang ${ }^{1,2}$, Bruce R. Southey ${ }^{2}$ and Sandra L. Rodriguez-Zas ${ }^{2,3,4^{*}}$ \\ ${ }^{1}$ Illinois Informatics Institute, University of Illinois at Urbana-Champaign, Urbana, IL, the U.S. \\ ${ }^{2}$ Department of Animal Sciences, University of Illinois at Urbana-Champaign, Urbana, IL, the \\ U.S. \\ ${ }^{3}$ Department of Statistics, University of Illinois at Urbana-Champaign, Urbana, IL, the U.S. \\ ${ }^{4}$ Carle Woese Institute for Genomic Biology, University of Illinois at Urbana-Champaign, \\ Urbana, IL, the U.S. \\ panz2@illinois.edu, southey@illinois.edu, rodrgzzs@illinois.edu
}

\begin{abstract}
Gene co-expression networks based on gene expression data are usually used to capture biologically significant patterns, enabling the discovery of biomarkers and interpretation of regulatory relationships. However, the coordination of numerous splicing changes within and across genes can exert a substantial impact on the function of these genes. This is particularly impactful in studies of the properties of the nervous system, which can be masked in the networks that only assess the correlation between gene expression levels. A bioinformatics approach was developed to uncover the role of alternative splicing and associated transcriptional networks using RNA-seq profiles. Data from 40 samples, including control and two treatments associated with sensitivity to stimuli across two central nervous system regions that can present differential splicing, were explored. The gene expression and relative isoform levels were integrated into a transcriptome-wide matrix, and then Graphical Lasso was applied to capture the interactions between genes and isoforms. Next, functional enrichment analysis enabled the discovery of pathways dysregulated at the isoform or gene levels and the interpretation of these interactions within a central nervous region. In addition, a Bayesian biclustering strategy was used to reconstruct treatment-specific networks from gene expression profile, allowing the identification of hub molecules and visualization of highly connected modules of isoforms and genes in specific conditions. Our bioinformatics approach can offer comparable insights into the discovery of biomarkers and therapeutic targets for a wide range of diseases and conditions.
\end{abstract}




\section{Introduction}

Gene co-expression networks are widely used to uncover the functionality of genes and interaction between genes at a system level. The constructed networks can enhance the understanding of the role and coordination of genes in diseases, treatments, environmental and physiological conditions, therefore advancing the understanding of molecular mechanisms underlying these conditions [1]. Most approaches used to reconstruct co-expression networks target gene relationships and use gene-level information. In these networks, nodes represent genes and edges reflect a high correlation between two genes based on the expression profile [2]. These approaches exclude information from alternative precursor-mRNA splicing, a critical post-transcriptional modification that can produce transcriptomes with distinct biological functions across tissues, brain regions, or other conditions [3]. Moreover, the disruption of splicing processes can play a significant role in the severity of diseases or impact of treatment, especially in processes that encompass the central nervous system.

Multiple studies have identified splicing variants involved in the incidence and severity of neuropathic pain [4-6]. However, the role on pain sensitivity of the transcript isoforms that result from alternative splicing events remain poorly understood $[6,7]$. Moreover, the typical study of transcript isoforms on an individual basis has the potential to hinder the detection of additional splicing variants and understanding of the interaction between these molecules and sensitivity to pain. RNA-seq profiling enables the study of the association between disorders such as sensitivity to pain both at the gene and transcript isoform levels. Bioinformatics approaches that mine the information available on the same samples at the gene and isoform levels can offer additional insights into the roles of both types of molecules on conditions such as sensitivity to pain. Furthermore, network inference approaches enhance the insights gained from the information available and could increase the power to detect novel alternative splicing isoforms and genes underlying diseases and disorders.

In the present work, a bioinformatics approach based on a network inference framework was used to integrate information at the isoform- and gene-levels on a transcriptome-wide basis. This strategy enabled us to systematically capture the relationships between splicing events on a set of genes and the expression of the remaining genes in the genome [8]. We demonstrate the capacity of this approach to uncover molecular relationships and build transcriptome-wide networks on multiple conditions and central nervous system regions. Next, Bayesian biclustering was used to identify condition-dependent relationships between genes [9]. We demonstrate the capacity of the proposed combination approaches to uncover relationships between transcript isoforms and genes that are unique or shared across conditions. The output from this study is an innovative bioinformatics strategy that could discover biomarkers and therapy targets for diseases and conditions.

\section{Methods}

\subsection{Expression data preparation}

The data used to demonstrate the capabilities of the bioinformatics approach proposed encompasses RNA-seq profiles from 40 samples from three conditions. These conditions include samples from two treatments associated with sensitivity to pain and corresponding control samples. One treatment influences pain sensation through the regulation of blood flow $(G)$ whereas the other treatment influences pain sensation through the receptors $(\mathrm{O})$. Furthermore, the samples represent central nervous system regions with known association to signaling and pain. One region is associated with sensing and processing of stimuli and pain $(\mathrm{T})$ and the other region is associated with processing of rewarding stimuli $(\mathrm{N})$. The RNA-seq profiles are available at the National Center for Biotechnology Information Gene Expression Omnibus (GEO) database, accession identifiers GSE126662 and GSE110194 [10, 11]. 
The raw sequencing data were aligned to the mouse reference genome (version GRCm38) using the STAR aligner (V2.5.3a) software [12]. The recorded expression levels were mapped to protein-coding genes and transcript isoforms using the GENCODE VM18 annotation [13]. Genes that were annotated to multiple Ensembl gene ID were removed. The levels of isoform and gene expression were quantified as raw reads count and Transcripts Per Kilobase Million (TPM) using RSEM (V1.3.1) [14].

\subsection{Gene-isoform networks based on Graphical Lasso}

A transcriptome-wide covariance matrix was estimated based on information on gene and transcript isoform expression levels. Recognizing the limitations of the absolute expression of a transcript, the transcript isoform information was analyzed on a relative expression level [8]. The genes and transcript isoforms that had more than 5 reads and with more than 1 TPM per treatment-region were further considered for the construction of the transcriptome-wide matrix. Gene and transcript isoform levels were log-transformed and normalized across samples. The dependency among isoform ratio patterns within a gene was resolved by excluding the isoform with the lowest ratio. The potential for an illconditioned expression matrix was minimized by using the top 3000 genes and top 4000 isoforms that had the highest variation to construct a transcriptome-wide matrix.

The relationships between genes and transcript isoforms were estimated using a scalable Graphical Lasso approach. The relationship among molecules was compiled into a sparse precision matrix $(\Theta)$ that is a function of the empirical covariance matrix (S) and of a matrix of penalties $(\lambda)$ including entries specific to edge types [15]:

$$
\widehat{\Theta}=\arg \min _{\Theta}\left\{\operatorname{tr}(\mathrm{S} \theta)-\log \operatorname{det} \Theta+|| \Lambda^{\circ} \Theta|| 1\right\}
$$

where $\Lambda$ is a matrix that has the same dimensions as $\Theta$, containing penalties for the interaction between modules. The glasso R package was used to obtain the network with a transcriptome-wide covariance matrix $\mathrm{S}$ and penalties matrix $\Lambda$ as input [16]. For the $i$ th row and $j$ th column of $\Lambda$,

$\begin{array}{lc}\text { if } \mathrm{i}=\mathrm{j}, & \lambda_{d} \text {; } \\ \text { if } \mathrm{i} \neq \mathrm{j} \text {, gene }(\mathrm{i})=\text { gene }(\mathrm{j}), & \lambda_{s} ; \\ \text { if gene }(\mathrm{i}) \neq \text { gene }(\mathrm{j}) \text {, type }(\mathrm{i})=\text { type }(\mathrm{j})=\text { gene, } & \lambda_{g g} \text {; } \\ \text { if gene }(\mathrm{i}) \neq \text { gene }(\mathrm{j}), \text { type }(\mathrm{i}) \text {, type }(\mathrm{j})\}=\{\text { gene, isoform }\}, & \lambda_{g i} \text {; } \\ \text { if gene }(\mathrm{i}) \neq \text { gene }(\mathrm{j}) \text {, type }(\mathrm{i})=\operatorname{type}(\mathrm{j})=\text { isoform, } & \lambda_{i i} \text {; }\end{array}$

where gene (x) denotes the gene corresponding to $x$ th molecule, and type (x) denotes the type of the $x$ th molecule (gene or isoform). The penalty assigned to the diagonal entries $\left(\lambda_{d}\right)$ of the precision matrix was set to 0 , and a penalty low in relation to the precision (0.05) was used for the off-diagonal elements representing different isoforms belonging to the same gene [17]. Other penalties were selected to achieve a scale-free network. The equation that describes the scale free property is equation $\mathrm{P}(\mathrm{k}) \sim \mathrm{k}^{-\mathrm{r}}$, $2<\mathrm{r}<3$, where $\mathrm{P}(\mathrm{k})$ is the fraction of nodes in a network that have $\mathrm{k}$ connections to other nodes, for large values of $\mathrm{k}$. The scale-free property was assessed using the correlation coefficient (cc) between $\log (\mathrm{P}(\mathrm{k}))$ and $\log (\mathrm{k})[18]$. Networks that had a $|\mathrm{cc}|>0.9$ were considered scale-free.

In the reconstructed precision matrix, non-zero entries represent an edge between two molecules (genes and isoforms). Edges that are not contribute to the biological interpretation of the relationships between genes, such as edges between two isoforms from the same gene, were removed.

\subsection{Treatment-specific gene networks based on Bayesian Biclustering}

Treatment-specific networks were inferred using an unsupervised Bayesian biclustering approach implemented using the software BicMix [14, 19]. The specification of the parameters for BicMix was 
based on results from simulation reports [20], for example, starting number of components is set as 100 . Genes that had less than 1 TPM or 6 reads in more than one treatment-region group were removed. Next, the TPM values within each gene were standardized and these normalized measurements were used as BicMix input. Normalized expression levels of 12492 genes from both nervous system regions were used within each treatment. BicMix was run 40 times with 300 iterations of the ExpectationMaximization algorithm within a run. For each run, co-expression network edges were extracted separately. Covariates matrices including condition information and other related effects were invented in order to identify the specific network type. The matrix is filled in with the corresponding value of the covariate for each sample. Edges supported by a probability higher than $80 \%$ within a run $[9,21]$, and edges detected in at least 10 runs (25\%) were depicted in the final treatment-specific networks. In this way, a specific network corresponding to a specific covariate with two different covariate types will be recovered. The software GeneNet was used to visualize the condition-specific networks [22].

\subsection{Functional enrichment analysis}

The identification of biological functions enriched among the highly connected nodes (hubs) from the transcriptome-wide networks and among the nodes of the condition-specific networks employed the web-service Database for Annotation, Visualization and Integrated Discovery, DAVID 6.8 [23]. The enrichment of Gene ontology (GO) categories and KEGG pathways in the DAVID database was evaluated using the Mus musculus genome as the background for enrichment testing. The enrichment was quantified using the Expression Analysis Systematic Explorer (EASE) score that is computed using a one-tailed jackknifed Fisher hypergeometric exact test. The enriched categories were clustered to facilitate interpretation, and the statistical significance of each cluster is evaluated as the geometric mean of the - $\log 10 \mathrm{EASE}$ scores of all the categories within the cluster [23].

\section{Results}

\subsection{Gene-isoform Graphical Lasso networks}

The output from the process that started with the estimation of a transcriptome-wide covariance matrix, application of scalable Graphical Lasso to obtain a precision matrix, and removal of edges with no biological support, were two transcriptome-wide networks. A scale-free network topology was achieved using penalty combinations $\lambda_{g g}=0.8, \lambda_{g i}=0.6, \lambda_{i i}=0.65$ for $\mathrm{N}$ and $\mathrm{T}$ networks including genes and isoforms. The correlation coefficient between $\log (\mathrm{P}(\mathrm{k}))$ and $\log (\mathrm{k})$ is -0.915 and -0.916 for $\mathrm{N}$ and T network respectively (Fig. 1).

The network for the $\mathrm{N}$ central nervous system region encompassed 6581 Gene-Gene edges, 9160 Gene-Isoform edges, and 3629 Isoform-Isoform edges, while in the network for the T central nervous system region included 3786 Gene-Gene edges, 9477 Gene-Isoform edges, and 3943 Isoform-Isoform edges. Four types of hubs corresponding to three edge types were identified: Gene-Gene hub, GeneIsoform hub, Isoform-Gene hub and Isoform-Isoform hub. 

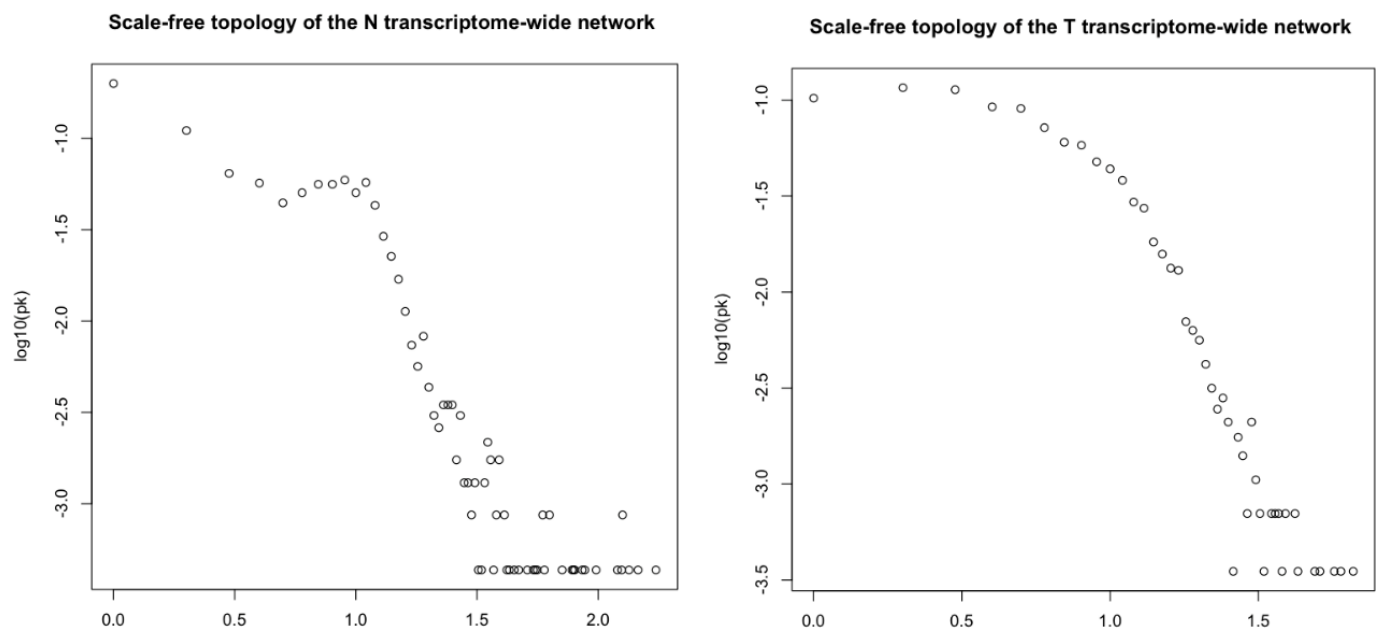

Fig. 1 Scale-free topology of the N (left) and T (right) transcriptome-wide networks

\subsection{Functional enrichments among hubs of Gene-Isoform networks}

The enrichment of Gene Ontology (GO) categories and KEGG pathways among the hubs of the final transcriptome-wide networks was assessed. The gene-isoform hub could assist with the identification of splicing regulators or modulators. Therefore, the gene-isoform hubs with a minimum of ten edges across the two transcriptome-wide networks were investigated using DAVID analysis.

Two clusters of RNA splicing-related categories with high DAVID enrichment scores (enrichment score $>1.2$ ) among 467 Gene-Isoform hubs of the transcriptome-wide network in the $\mathrm{N}$ region are presented in Table 1. Notable is the enrichment of Ribosome (KEGG mmu03010), structural molecule activity (GO:0005198), structural constituent of ribosome (GO:0003735), RNA binding (GO:0044822). Besides, GO term regulation of RNA splicing (GO:0043484) is significantly enriched with p value $<$ 0.05 and the involved genes are Serine/arginine-rich splicing factor 5 (Srsf5), Metastasis associated lung adenocarcinoma transcript 1 (Malat1), Nucleolysin TIA-1(Tia1), Heterogeneous nuclear ribonucleoproteins A2/B1(Hnrnpa2b1), Dual specificity protein kinase CLK4 (Clk4), Dual specificity protein kinase CLK1 (Clk1), Heat shock cognate $71 \mathrm{kDa}$ protein (Hspa8).

Table 1. Clusters of enriched functional categories (enrichment score ES > 1.2) related with RNA splicing among the Gene-Isoform hub in the $\mathrm{N}$ transcriptome-wide network using DAVID

\begin{tabular}{llll}
\hline Category & Category identifier and name & P value & FDR P value \\
\hline Cluster 1 & ES $=2.34$ & & \\
KEGG & mmu03010:Ribosome & $8.5 \mathrm{E}-07$ & $5.1 \mathrm{E}-05$ \\
MF & GO:0005198 structural molecule activity & $6.7 \mathrm{E}-04$ & $1.6 \mathrm{E}-02$ \\
MF & GO:0003735 structural constituent of ribosome & $6.8 \mathrm{E}-04$ & $1.5 \mathrm{E}-02$ \\
Cluster 2 & ES $=1.28$ & & \\
MF & GO:0044822 poly(A) RNA binding & $4.6 \mathrm{E}-05$ & $2.0 \mathrm{E}-03$ \\
MF & GO:0003723 RNA binding & $9.9 \mathrm{E}-04$ & $2.0 \mathrm{E}-02$ \\
\hline
\end{tabular}


The most significant DAVID clusters of functional categories enriched among the 373 Gene-Isoform hubs of the $\mathrm{T}$ transcriptome-wide network are listed in Table 2. Informative clusters of enriched categories include the terms: Ribosome and structural molecule activity (KEGG mmu03010 and GO:0005198); poly(A) RNA binding and RNA binding (GO:0048255 and GO:0003723); mRNA stabilization and RNA stabilization (GO:0048255 and GO:0043489); regulation of RNA splicing, mRNA binding, RNA processing and RNA splicing (GO:0043484, GO:0003729, GO:0006396 and GO:0008380). Genes Short stature homeobox protein 2 (Shox2), KH domain-containing, RNA-binding, signal transductionassociated protein 1 (Khdrbs1), Serine/arginine-rich splicing factor 2 (Srsf2), Heterogeneous nuclear ribonucleoprotein $\mathrm{H}$ (Hnrnph1), AHNAK nucleoprotein (Ahnak), Phosphatidylinositol 3-kinase regulatory subunit alpha (Pik3r1), DNA-directed RNA polymerase II subunit RPB1 (Polr2a) along with Srsf5, malat1, Hnrnp2b1 and Clk1 contribute to the enrichment of regulation of RNA splicing.

Apart from the RNA splicing related enrichments, KEGG pathways Glutamatergic synapse (mmu04724), GABAergic synapse (mmu04727), Retrograde endocannabinoid signaling (mmu04723) and GO term regulation of sensory perception of pain (GO:0051930) are also enriched among Gene-Isoform hubs of the N transcriptome-wide network. And among GeneIsoform hubs of the $\mathrm{T}$ transcriptome-wide network, response to pain (GO:0048265), sensory perception of pain (GO:0019233).

Table 2. Clusters of enriched functional categories (enrichment score ES > 1.5) related with RNA splicing among the Gene-Isoform hub in the T transcriptome-wide network using DAVID

\begin{tabular}{llll}
\hline Category & Category identifier and name & P value & FDR P value \\
\hline Cluster 1 & ES $=4.69$ & & \\
MF & GO:0005198 structural molecule activity & $6.80 \mathrm{E}-09$ & $2.90 \mathrm{E}-06$ \\
KEGG & mmu03010:Ribosome & $4.80 \mathrm{E}-06$ & $4.60 \mathrm{E}-04$ \\
MF & GO:0003735 structural constituent of ribosome & $6.00 \mathrm{E}-04$ & $1.80 \mathrm{E}-02$ \\
Cluster 2 & ES $=3.74$ & & \\
MF & GO:0044822 poly(A) RNA binding & $2.40 \mathrm{E}-09$ & $2.00 \mathrm{E}-06$ \\
MF & GO:0003723 RNA binding & $1.80 \mathrm{E}-07$ & $3.90 \mathrm{E}-05$ \\
Cluster 3 & ES $=1.96$ & & \\
BP & GO:0048255 mRNA stabilization & $3.00 \mathrm{E}-03$ & $4.30 \mathrm{E}-02$ \\
Cluster 4 & ES $=1.64$ & & \\
BP & GO:0043484 regulation of RNA splicing & $1.60 \mathrm{E}-05$ & $7.90 \mathrm{E}-04$ \\
MF & GO:0003729 mRNA binding & $1.90 \mathrm{E}-03$ & $4.30 \mathrm{E}-02$ \\
\hline
\end{tabular}

\subsection{Biclustering networks}

Further exploration of treatment-specific co-expression relationships on pain-sensitivity treatments was gained from the Bayesian biclustering analysis. For the $\mathrm{O}$ treatment network, 43 edges among 23 genes are uniquely identified in this treatment condition and the genes in the network are highly 
connected (Fig. 2). Among these genes, solute carrier family 1 member 6 (Slc1a6), Sodium VoltageGated Channel Alpha Subunit 5 (Scn5a), Protein NYNRIN (Nynrin), Coiled-coil domain-containing protein 184 (Ccdc184) and Glutamate receptor ionotropic, NMDA 2D (Grin2d) have the highest degree. Results from the DAVID analysis identified the enrichment of the Glutamatergic synapse pathway, regulation of membrane potential, and nitrogen compound metabolic process.

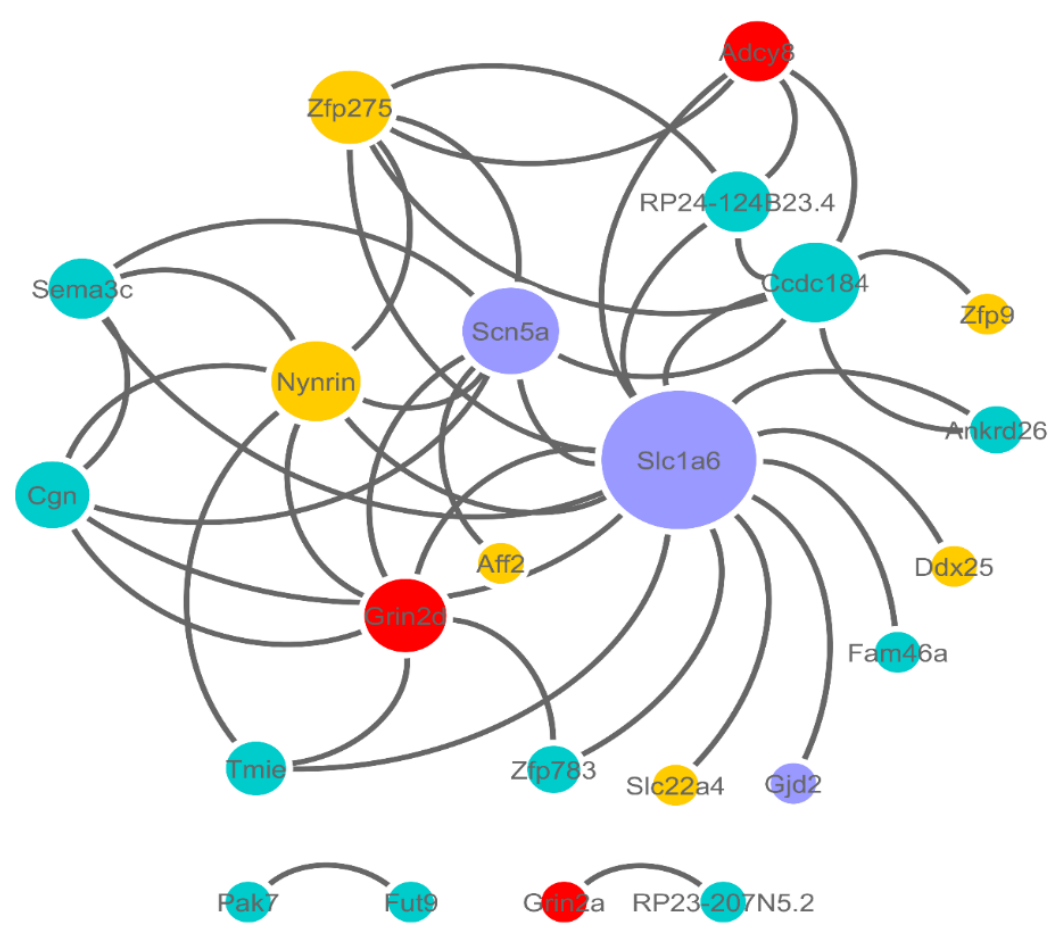

Fig. 2 Biclustering network of the receptor-based treatment $\mathrm{O}$. Node size reflects betweenness centrality of the nodes. Node color: genes enriched in Glutamatergic synapse pathway are depicted in red; genes enriched in regulation of membrane potential BP GO term are depicted in purple; genes in nitrogen compound metabolic process are depicted in yellow.

\section{Discussion}

The bioinformatics approach used in this study enabled the reconstruction of co-expression networks among genes and transcript isoforms and the discovery of molecular mechanisms associated with sensitivity to stimuli. Multiple studies reported that alternative splicing is a fundamental approach for a neuronal cell to regulate specific biological functions [24]. The transcriptome-wide framework that includes gene expression and isoform relative abundance enabled the detection of splicing regulation and interplay between genes and isoforms, which is supported by the enriched regulation of RNA splicing (GO:0043484) and RNA splicing (GO:0008380) categories. An interesting finding from the gene-isoform Graphical Lasso analysis is the enrichment of the Ribosome (KEGG mmu03010), structural constituent of ribosome (GO:0003735), structural molecule activity (GO:0005198) and RNA binding (GO:0003723) categories among the Gene-Isoform hubs in both regions associated with 
response to pain and to reward stimuli. That is in agreement with the reports that the ribosome and RNA binding is engaged in alternative splicing events [25-27]. Also, the enriched categories such as Glutamatergic synapse, GABAergic synapse and Retrograde endocannabinoid signaling are consistent with reports of the association of these pathways and neuropathic pain $[11,28]$. In addition, the enrichment of response to pain, regulation of sensory perception of pain and sensory perception of pain are in accordance with two sensitivity to stimuli associated treatments.

Among the results from the treatment-specific co-expression networks identified using the Bayesian biclustering approach, relationships unique to the treatments studied were detected. For the receptor treatment $\mathrm{O}$, genes in the network are annotated to the Glutamatergic synapse pathway, and it is in accordance with previous reports $[10,29]$. Among the genes that exhibited a high degree treatment specificity, the function of Nav1.5 channel gene SCN5A in neuropathic pain and migraine has been reported [30, 31]. Further exploration of the networks of additional pain sensitivity-related treatments will advancing the understanding of the molecular mechanism underlying these conditions.

In summary, the reconstruction of transcriptome wide networks together with treatment-, conditionor disease-specific network inference can provide a comprehensive map of splicing regulation, gene interaction, and co-expression corresponding to the status. This approach can offer novel insights into the discovery of biomarkers and therapeutic targets for a wide range of diseases and conditions.

\section{Acknowledgements}

This study was funded by the National Institute of Health (grant numbers P30 DA018310-14, and DA031243 (AP)), the Department of Defense (grant number PR100085), and China Scholarship Council, CSC NO. 201606140027.

We are grateful to Dr. A. Pradhan and J. Sweedler for conceptualization of the study, sample collection, and provision of resources.

\section{Reference}

[1] Yang, Y., et al., Gene co-expression network analysis reveals common system-level properties of prognostic genes across cancer types. Nat Commun, 2014. 5: p. 3231.

[2] Zhang, B. and S. Horvath, A general framework for weighted gene co-expression network analysis. Stat Appl Genet Mol Biol, 2005. 4: p. Article17.

[3] Matlin, A.J., F. Clark, and C.W. Smith, Understanding alternative splicing: towards a cellular code. Nat Rev Mol Cell Biol, 2005. 6(5): p. 386-98.

[4] Warner, S.C., et al., Genome-wide association scan of neuropathic pain symptoms post total joint replacement highlights a variant in the protein-kinase C gene. Eur J Hum Genet, 2017. 25(4): p. 446-451.

[5] Marchi, M., et al., A novel SCN9A splicing mutation in a compound heterozygous girl with congenital insensitivity to pain, hyposmia and hypogeusia. J Peripher Nerv Syst, 2018. 23(3): p. 202-206.

[6] Hulse, R.P., et al., The control of alternative splicing by SRSF1 in myelinated afferents contributes to the development of neuropathic pain. Neurobiol Dis, 2016. 96: p. 186-200.

[7] Ahmed, M.M., et al., Pathogenesis of spinal cord injury induced edema and neuropathic pain: expression of multiple isoforms of wnkl. Ann Neurosci, 2014. 21(3): p. 97-103.

[8] Saha, A., et al., Co-expression networks reveal the tissue-specific regulation of transcription and splicing. Genome Res, 2017. 27(11): p. 1843-1858. 
[9] Gao, C., et al., Context Specific and Differential Gene Co-expression Networks via Bayesian Biclustering. PLoS Comput Biol, 2016. 12(7): p. e1004791.

[10] Zhang, P., et al., Opioid-Induced Hyperalgesia Is Associated with Dysregulation of Circadian Rhythm and Adaptive Immune Pathways in the Mouse Trigeminal Ganglia and Nucleus Accumbens. Mol Neurobiol, 2019.

[11] Jeong, H., et al., Gene Network Dysregulation in the Trigeminal Ganglia and Nucleus Accumbens of a Model of Chronic Migraine-Associated Hyperalgesia. Front Syst Neurosci, 2018. 12: p. 63.

[12] Dobin, A., et al., STAR: ultrafast universal RNA-seq aligner. Bioinformatics, 2013. 29(1): p. 15-21.

[13] Frankish, A., et al., GENCODE reference annotation for the human and mouse genomes. Nucleic Acids Res, 2019. 47(D1): p. D766-D773.

[14] Li, B. and C.N. Dewey, RSEM: accurate transcript quantification from RNA-Seq data with or without a reference genome. BMC Bioinformatics, 2011. 12: p. 323.

[15] Friedman, J., T. Hastie, and R. Tibshirani, Sparse inverse covariance estimation with the graphical lasso. Biostatistics, 2008. 9(3): p. 432-41.

[16] Hsieh, C.J., et al., QUIC: Quadratic Approximation for Sparse Inverse Covariance Estimation. Journal of Machine Learning Research, 2014. 15: p. 2911-2947.

[17] Saha, A., et al., Co-expression networks reveal the tissue-specific regulation of transcription and splicing. Genome Research, 2017. 27(11): p. 1843-1858.

[18] Zhang, B. and S. Horvath, A general framework for weighted gene co-expression network analysis. Statistical Applications in Genetics and Molecular Biology, 2005. 4.

[19] Gao, C., et al., Context Specific and Differential Gene Co-expression Networks via Bayesian Biclustering. Plos Computational Biology, 2016. 12(7).

[20] Saengtrakul, K., S. Kanjanawasee, and N. Wiratchai, Student factors affecting latent transition of mathematics achievement measuring from latent transition analysis with a mixture item response theory measurement model. Future Academy Multidisciplinary Conference Iceepsy \& Cpsyc \& Icpsirs \& Be-Ci, 2016. 217: p. 729-737.

[21] Schafer, J. and K. Strimmer, An empirical Bayes approach to inferring large-scale gene association networks. Bioinformatics, 2005. 21(6): p. 754-64.

[22] Ananko, E.A., et al., GeneNet: a database on structure and functional organisation of gene networks. Nucleic Acids Res, 2002. 30(1): p. 398-401.

[23] Huang da, W., B.T. Sherman, and R.A. Lempicki, Systematic and integrative analysis of large gene lists using DAVID bioinformatics resources. Nat Protoc, 2009. 4(1): p. 44-57.

[24] Saito, Y., et al., Differential NOVA2-Mediated Splicing in Excitatory and Inhibitory Neurons Regulates Cortical Development and Cerebellar Function. Neuron, 2019. 101(4): p. 707-720 e5.

[25] Weatheritt, R.J., T. Sterne-Weiler, and B.J. Blencowe, The ribosome-engaged landscape of alternative splicing. Nat Struct Mol Biol, 2016. 23(12): p. 1117-1123.

[26] Furlanis, E., et al., Landscape of ribosome-engaged transcript isoforms reveals extensive neuronal-cell-class-specific alternative splicing programs. Nat Neurosci, 2019. 22(10): p. 1709-1717.

[27] Lee, Y. and D.C. Rio, Mechanisms and Regulation of Alternative Pre-mRNA Splicing. Annu Rev Biochem, 2015. 84: p. 291-323.

[28] Du, X., et al., Local GABAergic signaling within sensory ganglia controls peripheral nociceptive transmission. J Clin Invest, 2017. 127(5): p. 1741-1756.

[29] Bie, B. and Z.Z. Pan, Presynaptic mechanism for anti-analgesic and anti-hyperalgesic actions of kappa-opioid receptors. J Neurosci, 2003. 23(19): p. 7262-8. 
[30] Aromolaran, A.S., M. Chahine, and M. Boutjdir, Regulation of Cardiac Voltage-Gated Sodium Channel by Kinases: Roles of Protein Kinases A and C. Handb Exp Pharmacol, 2018. 246: p. 161-184.

[31] Wang, J., et al., Downregulation of adult and neonatal Nav1.5 in the dorsal root ganglia and axon of peripheral sensory neurons of rats with spared nerve injury. Int J Mol Med, 2018. 41(4): p. 2225-2232. 